\title{
Universal approach to overcoming nonstationarity, unsteadiness and non-Markovity of stochastic processes in complex systems
}

\author{
Renat M. Yulmetyev ${ }^{\mathrm{a}, *}$, Anatolii V. Mokshin ${ }^{\mathrm{a}}$, Peter Hänggi ${ }^{\mathrm{b}}$ \\ ${ }^{a}$ Department of Theoretical Physics, Kazan State Pedagogical University, Kazan, Mezhlauk 1, \\ Kazan 420021, Russia \\ ${ }^{\mathrm{b}}$ Department of Physics, University of Augsburg, Universitätsstrasse 1, D-86135 Augsburg, Germany
}

Received 12 September 2003

Available online 3 August 2004

\begin{abstract}
In the present paper, we suggest a new universal approach to study complex systems by microscopic, mesoscopic and macroscopic methods. We discuss new possibilities of extracting information on nonstationarity, unsteadiness and non-Markovity of discrete stochastic processes in complex systems. We consider statistical properties of the fast, intermediate and slow components of the investigated processes in complex systems within the framework of microscopic, mesoscopic and macroscopic approaches separately. Among them theoretical analysis is carried out by means of local noisy time-dependent parameters and the conception of a quasi-Brownian particle (QBP) (mesoscopic approach) as well as the use of wavelet transformation of the initial row time series. As a concrete example we examine the seismic time series data for strong and weak earthquakes in Turkey $(1998,1999)$ in detail, as well as technogenic explosions. We propose a new possible solution to the problem of forecasting strong earthquakes. Besides we have found out that an unexpected restoration of the first two local noisy parameters in weak earthquakes and technogenic explosions is determined by exponential law. In this paper we have also carried out the comparison and
\end{abstract}

\footnotetext{
*Corresponding author. Tel.: + 7-8432-925373; fax: + 7-8432-924269.

E-mail address: rmy@theory.kazan-spu.ru (R.M. Yulmetyev).
} 
have discussed the received time dependence of the local parameters for various seismic phenomena.

(C) 2004 Elsevier B.V. All rights reserved.

PACS: 02.50.Ey; 05.45.Tp; 89.75.-k

Keywords: Stochastic processes; Complex systems; Non-Markovity; Localization procedure; Nonstationarity; Unsteadiness

\section{Introduction}

Nonstationarity, unsteadiness and non-Markovity are the most common essential peculiarities of stochastic processes in nature. The existence of similar properties creates significant difficulties for the theoretical analysis of real complex systems [1]. At present, methods connected with localization of the registered or calculated parameters for the quantitative account of the dramatic changes caused by the fast alternation of the behavior modes and intermittency came into use. For example, the time behavior of the local (scale) Hurst exponents was found in the recent work of Stanley et al. to study multifractal cascades in heartbeat dynamics [1] and to analyze and forecast earthquakes and technogenic explosions in Ref. [2]. The application of the local characteristics allows to avoid difficulties connected with nonergodicity of the investigated system and gives a possibility to extract additional valuable information on the hidden properties of real complex systems. From the physical point of view this approach resembles the use of nonlinear equations of generalized hydrodynamics with the local time behavior of hydrodynamical and thermodynamical parameters and characteristics.

It is well known that one of the major problems of seismology is to predict the beginning of the main shock. Although science still seems to be far from the guaranteed decision of this problem there exist some interesting approaches based on the peculiar properties of precursory phenomena [3-9]. Another important problem is recognition and differentiation of weak earthquakes and technogenic underground explosion signals. One of the useful means of solving this problem is by defining their local characteristics $[1,2]$.

In the present work we suggest a new universal description of real complex systems by means of the microscopic, mesoscopic and macroscopic methods. We start with a macroscopic approach based on the kinetic theory of discrete stochastic processes and the hierarchy of the chain of finite-difference kinetic equations for the discrete time correlation function (TCF) and memory functions [2,10,11].

The mesoscopic phenomena of the so-called "soft matter" physics, embracing a diverse range of system including liquid crystals, colloids, and biomembranes, generally involve some form of coupling of different characteristic time- and lengthscales. Computational modelling of such multi-scale effects requires a new methodology applicable beyond the realm of traditional techniques such as $a b$ initio and classical molecular dynamics (the methods of choice in the microscopic 
regime), as well as phase field modelling or the lattice-Boltzmann method (usually concerned with the macroscopic regime).

We propose to consider intermediate and slow processes within a unified framework of mesoscopic approach: by means of local time behavior of the local relaxation and kinetic parameters, local non-Markovity parameters and so on. For this purpose we introduce the notion of quasi-Brownian motion in a complex system by coarse-grained averaging of the initial time series on the basis of wavelet transformation.

As an example we consider here the local properties of relaxation or noise parameters for the analysis of seismic phenomena such as earthquakes and technogenic explosions. The layout of the paper is as follows. In Section 2 we describe in brief the stochastic dynamics of time correlation in complex systems containing seismic signals by means of discrete non-Markov kinetic equations. The basic equations used for these calculations are presented here. The local noise parameters are defined in Section 3. Section 4 contains the results obtained by the local noise parameter procedure. The models of the time dependence of the local parameters are given in Section 5. The basic conclusions are discussed in Section 6.

\section{The basic definitions in kinetic description of discrete stochastic processes}

\subsection{Macroscopic description in the analysis of stochastic processes}

Several different existent processes, such as economical, meteorogical, gravimetrical and other data are registered as discrete random series $x_{i}$ of some variable $X$. This random variable $X$ can be written as an array of its values

$$
X=\{x(T), x(T+\tau), x(T+2 \tau), \ldots, x(T+k \tau), \ldots, x(T+(N-1) \tau)\}
$$

Here a time step (or a time interval) $\tau$ is a constant, $T$ is the time when the registration of the signal begins, and $(N-1) \tau$ is the duration of the signal detection.

The average value $\langle x\rangle$ and fluctuations $\delta x_{j}$ are defined by the following expressions, correspondingly:

$$
\langle x\rangle=\frac{1}{N} \sum_{j=0}^{N-1} x(T+j \tau), \quad \delta x_{j}=\delta x(T+j \tau)=x(T+j \tau)-\langle x\rangle .
$$

From the fluctuations of the considered random variable $\delta x_{j}$ we can form $k$ component state vector of the system's correlation state

$$
\begin{aligned}
\mathbf{A}_{k}^{0} & =\mathbf{A}_{k}^{0}(0)=(\delta x(T), \delta x(T+\tau), \ldots, \delta x(T+(k-1) \tau)) \\
& =\left(\delta x_{0}, \delta x_{1}, \ldots, \delta x_{k-1}\right)
\end{aligned}
$$


The time dependence of the correlation state vector $\mathbf{A}$ can be represented as a discrete $m$-step shift

$$
\begin{aligned}
\mathbf{A}_{m+k}^{m} & =\mathbf{A}_{m+k}^{m}(t)=(\delta x(T+m \tau), \delta x(T+(m+1) \tau), \ldots, \delta x(T+(m+k+1) \tau)) \\
& =\left(\delta x_{0}, \delta x_{1}, \ldots, \delta x_{m+k-1}\right)
\end{aligned}
$$

Then by analogy with the papers $[2,10,11]$ we can write the following normalized TCF:

$$
\begin{aligned}
M_{0}(t) & =\frac{\left\langle\mathbf{A}_{N-1-m}^{0} \mathbf{A}_{N-1}^{m}\right\rangle}{\left\langle\mathbf{A}_{N-1-m}^{0} \mathbf{A}_{N-1-m}^{0}\right\rangle}=\frac{\left\langle\mathbf{A}_{N-1-m}^{0}(0) \mathbf{A}_{N-1}^{m}(t)\right\rangle}{\left|\mathbf{A}_{N-1-m}^{0}(0)\right|^{2}} \\
& =\frac{\left\langle\mathbf{A}_{N-1-m}^{0}(0) U(t=T+m \tau, T) \mathbf{A}_{N-1-m}^{0}(0)\right\rangle}{\left|\mathbf{A}_{N-1-m}^{0}(0)\right|^{2}}
\end{aligned}
$$

where the angular brackets indicate the scalar product of the two state vectors. On the other hand, the time dependence of vector $\mathbf{A}_{N-1-m}^{m}(T+t), t=m \tau$, can be represented formally with the help of the evolution operator $U\left(t^{\prime}, t\right)$ as follows:

$$
\mathbf{A}_{N-1}^{m}(T+t)=U(T+m \tau, T) \mathbf{A}_{N-1-m}^{0}(T)=U(t, 0) \mathbf{A}_{N-1-m}^{0}(0) .
$$

The last one has the property: $U(t, t)=1$. Actually, one can write down formal discrete equation of motion with the use of the evolution operator $U\left(t^{\prime}, t\right)$ (see Appendix A for more details).

It was shown in Refs. $[2,10,11]$ that the finite-difference kinetic equation of a nonMarkov type for TCF $M_{0}(t)$ can be written by means of the technique of projection operators of Zwanzig'-Mori's type [14,15]

$$
\frac{\Delta M_{0}(t)}{\Delta t}=\lambda_{1} M_{0}(t)-\tau \Lambda_{1} \sum_{j=0}^{m-1} M_{1}(j \tau) M_{0}(t-j \tau) .
$$

Here the first-order memory function $M_{1}(j \tau)$ appears, $\lambda_{1}$ is an eigenvalue of Liouville's quasioperator $\hat{\mathscr{L}}$ (see Appendix A) and $\Lambda_{1}$ is a relaxation noise parameter, which are characteristics of the investigated process. Possible methods of defining quasioperator $\hat{\mathscr{L}}$ are presented in Appendix A [see Eqs. (A.3), (A.5) and (A.7)]. It should be noted that Eq. (7) is the first kinetic finite-difference equation for initial TCF $M_{0}(t)$. With the use of the same procedure of projection operator we can obtain the chain of kinetic finite-difference equations of the form

$$
\frac{\Delta M_{i-1}(t)}{\Delta t}=\lambda_{i} M_{i-1}(t)-\tau \Lambda_{i} \sum_{j=0}^{m-1} M_{i}(j \tau) M_{i-1}(t-j \tau), \quad i=1,2,3, \ldots
$$

Here $\lambda_{i}$ and $\Lambda_{i}$ are noise parameters, and $M_{i}(j \tau)$ is the memory function of the $i$ th order

$$
\lambda_{n}=i \frac{\left\langle\mathbf{W}_{n-1} \hat{\mathscr{L}} \mathbf{W}_{n-1}\right\rangle}{\left|\mathbf{W}_{n-1}\right|^{2}} ; \quad \Lambda_{n}=i \frac{\left\langle\mathbf{W}_{n-1} \hat{\mathscr{L}} \mathbf{W}_{n}\right\rangle}{\left|\mathbf{W}_{n-1}\right|^{2}}
$$


Here $\mathbf{W}_{n}$ are the dynamical orthogonal variables, obtained by the Gram-Schmidt orthogonalization procedure

$$
\left\langle\mathbf{W}_{n}, \mathbf{W}_{m}\right\rangle=\delta_{n, m}\left\langle\left|\mathbf{W}_{n}\right|^{2}\right\rangle,
$$

where $\delta_{n, m}$ is the Kronecker's symbol,

$$
\begin{aligned}
& \mathbf{W}_{0}=\mathbf{A}_{k}^{0}(0), \quad \mathbf{W}_{1}=\left[i \hat{\mathscr{L}}-\lambda_{1}\right] \mathbf{W}_{0}, \\
& \mathbf{W}_{2}=\left[i \hat{\mathscr{L}}-\lambda_{2}\right] \mathbf{W}_{1}-\Lambda_{1} \mathbf{W}_{0}, \ldots .
\end{aligned}
$$

From Eq. (10) it is obvious that in the cited Gram-Schmidt procedure from each new vector of state one should subtract the projection on all the previous vectors. Thereafter the orthogonalization (10) is complete.

A chain of integro-differential equations (8) arise as a result of the use of projection operator technique to define different correlation functions in physical problems [14,15] (for example, TCF of density fluctuation in inelastic neutron scattering [12,13] and light scattering investigations, velocity autocorrelation function and others can be received in a specified way). However, the quest for a physically based way of closing the chain of equations and finding the spectra of the initial TCF are essential moments in these challenges. Here the situation is different. Namely, the initial TCF can be calculated directly from the experimental data. Then memory functions $M_{i}$ and noise parameters $\lambda_{i}, \Lambda_{i}$ are similarly calculated from the experiment. All these functions and parameters make it possible to carry out the detailed analysis of the random process. The noise parameters $\lambda_{i}$ and $\Lambda_{i}$ are the relaxation characteristics of the experimental time series, which contain information of various modes passing and changing. The macroscopic approach presented above is based on the calculation of memory functions, power spectra, dynamic variables, and relaxation parameters. It suggests the investigation of the system as a single whole. The global characteristics calculated on the basis of all the time series contain hidden information about various modes of the system behavior. As a rule, this information is difficult to extract and analyze. For this reason it is necessary to develop a mesoscopic description and introduce a local noise and relaxation parameters $\lambda_{i}$ and $\Lambda_{i}$.

\subsection{Mesoscopic description in analysis of stochastic processes}

It is well known that the mesoscopic conception is one of the possible ways to deal with random processes in complex systems. It consists of extension of the domain of the dynamical equations mesoscopic variables and of introduction of some local time interval. Such a quantity as $X$ (it can be particle position, mass density and so on) is defined on the mesoscopic space [20]. In addition we introduce number $M$ as its extensive quantity. This number $M$ should satisfy the condition

$$
1 \ll M \ll N \text {, }
$$

where $N$ is the length of the initial experimental sampling. Let us take a working window of the fixed length $M$. By superposing this window on the initial sampling $X$, 
we choose all elements, incoming, as a separate sampling $\bar{\xi}_{0}$. Further, let us execute one time step $\tau$ shift of this working window to the right and obtain another local sampling of length $M$. Executing this procedure $(N-M+1)$ times, one can obtain the same quantity of the local samplings of length $M$ :

$$
\begin{aligned}
\bar{\xi}_{0}= & \bar{\xi}_{0}\{x(T), x(T+\tau), x(T+2 \tau), \ldots, x(T+(M-1) \tau)\}, \\
\bar{\xi}_{1}= & \bar{\xi}_{1}\{x(T+\tau), x(T+2 \tau), x(T+3 \tau), \ldots, x(T+M \tau)\}, \\
& \vdots \\
\bar{\xi}_{N-M}= & \bar{\xi}_{N-M}\{x(T+(N-M-1) \tau), x(T+(N-M) \tau), \\
& x(T+(N-M+1) \tau) \ldots, x(T+(N-1) \tau)\} .
\end{aligned}
$$

In its turn the obtained local samplings $\bar{\xi}_{i}$ form the array, which represents the time dynamics of the investigated process

$$
\bar{\xi}\left(t^{\prime}\right)=\left\{\bar{\xi}_{0}, \bar{\xi}_{1}, \bar{\xi}_{2}, \ldots, \bar{\xi}_{i}, \ldots, \bar{\xi}_{N-M}\right\}
$$

Then in accordance with the procedure described in the last Section 2.1, we can define fluctuations by Eq. (2), calculate TCF with the help of Eq. (5), and calculate memory functions and parameters $\lambda_{i}$ and $\Lambda_{i}$ by Eqs. (9) for every sampling $\bar{\xi}_{i}$. However, parameters $\lambda_{i}$ and $\Lambda_{i}$ will be characteristic of concrete $j$ th sampling only. Thus, they will characterize the local properties of the initial time data $X$ and will change at cross-over from one local sampling $\bar{\xi}_{i}$ to another $\bar{\xi}_{i+1}$. Then it is convenient to represent their local time dependence in the following way:

$$
\begin{aligned}
\lambda_{i}\left(t^{\prime}\right)= & \left\{\lambda_{i}(T+(M-1) \tau), \lambda_{i}(T+M \tau),\right. \\
& \left.\lambda_{i}(T+(M+1) \tau), \ldots, \lambda_{i}(T+(N-M) \tau)\right\}, \\
\Lambda_{i}\left(t^{\prime}\right)= & \left\{\Lambda_{i}(T+(M-1) \tau), \Lambda_{i}(T+M \tau),\right. \\
& \left.\Lambda_{i}(T+(M+1) \tau), \ldots, \Lambda_{i}(T+(N-M) \tau)\right\} .
\end{aligned}
$$

Operating in a similar way, one can execute cross-over from the macroscopic description of the whole system to the mesoscopic one. The offered approach is very convenient for the description and analysis of nonstationary stochastic processes. It allows to depart from the global macro-characteristics, which carry only averaged minor information about the whole investigated process, and to turn to the stochastic description with the use of the local characteristics and to execute a more detailed analysis of various dynamic states of the system.

\subsection{Conception of one-dimensional dynamics of quasi-Brownian particle}

Let us consider the motion of a large Brownian particle in a dense medium composed of light molecules. Let us restrict it by a simple one-dimensional case. Coordinate $x_{i}$ and velocity $v_{i}$ are random variables of a Brownian particle. Quantity $\tau$ represents the average time between the two successive collisions of liquid molecules; $T$ is the initial moment of time. Variable $M$ characterizes a local size (mass) of a Brownian particle. It is obvious, that a Brownian particle must have a 
larger mass in comparison with liquid particles $(M \gg 1)$; therefore, it is more inert. Then it is convenient to define the coordinate of a Brownian particle at moment $t^{\prime}$ as an average value of sampling $\bar{\xi}_{i}$. For example, we obtain from $\bar{\xi}_{0}$

$$
\begin{aligned}
y_{0} & =\frac{x(T)+x(T+\tau)+x(T+2 \tau)+\cdots+x(T+(M-1) \tau)}{M} \\
& =\frac{1}{M} \sum_{j=0}^{M-1} x(T+j \tau) .
\end{aligned}
$$

Quantity $y_{0}$ defines the coordinate of "the center of gravity" of a Brownian particle at the initial time moment $t^{\prime}=0$. By analogy it can define the position of a Brownian particle at the next time moment $t^{\prime}=\tau$ and so on. As a result we obtain a new time discrete series $Y\left(t^{\prime}\right)$ as

$$
Y\left(t^{\prime}\right)=\left\{y_{0}, y_{1}, y_{2}, \ldots, y_{N-M}\right\} .
$$

The velocity of a Brownian particle is

$$
v_{i}=\frac{y_{i+1}-y_{i}}{\tau} .
$$

Thus, for example, for the initial velocity $v_{0}$ we obtain from Eqs. (12), (15) and (17):

$$
v_{0}=\frac{y_{1}-y_{0}}{\tau}=\frac{x(T+M \tau)-x(T)}{M \tau} .
$$

Obviously, if the larger one is $M$, the smaller one is the velocity of a Brownian particle. Hence, we obtain a discrete set of velocity values for a Brownian particle at an equally small time interval $\tau$

$$
V\left(t^{\prime}\right)=\left\{v_{0}, v_{1}, v_{2}, \ldots, v_{N-M-1}\right\} .
$$

Eqs. (16) and (19) define time dependence of random variables $Y\left(t^{\prime}\right)$ and $V\left(t^{\prime}\right)$.

\subsubsection{Generalized Langevin equation with discrete time}

In accordance with Eq. (2), let us define the correlation state vector, whose components are the fluctuations of the particle position

$$
\mathbf{B}_{k}^{0}=\left\{\delta y_{0}, \delta y_{1}, \delta y_{3}, \ldots, \delta y_{k-1}\right\} .
$$

Then by analogy to (4) time dependence of vector $\mathbf{B}$ can be considered a discrete $m$ step time shift. For vector $\mathbf{B}$ the following normalized TCF can be written with the help of Eq. (5):

$$
b(t)=\frac{\left\langle\mathbf{B}_{N-1-m}^{0} \mathbf{B}_{N-1}^{m}\right\rangle}{\left|\mathbf{B}_{N-1-m}^{0}\right|^{2}} .
$$

The last one describes the time correlation of the two different correlation states of the system. 
Now let us introduce the linear projection operator in Euclidean space of the state vectors:

$$
\left.Q \mathbf{B}=\frac{\mathbf{B}(0)\rangle\langle\mathbf{B}(0) \mathbf{B}(t)\rangle}{|\mathbf{B}(0)|^{2}}=\mathbf{B}(0)\right\rangle b(t), \quad Q=\frac{\mathbf{B}(0)\rangle\langle\mathbf{B}(0)}{\langle\mathbf{B}(0) \mathbf{B}(0)\rangle} .
$$

This operator has the necessary property of idempotency $Q^{2}=Q$. The existence of projection operator $Q$ allows to introduce the mutually supplementary projection operator $R$ as follows:

$$
R=1-Q, \quad R^{2}=R, \quad Q R=R Q=0 .
$$

It is necessary to note that projectors $Q$ and $R$ are both linear and can be recorded for the fulfillment of projection operations in the particular Euclidean space. The projection operators $Q$ and $R$ allow to carry out the splitting of Euclidean space of vectors $B$, where $B(0)$ and $B(t) \in B$, into a straight sum of the two mutually supplementary subspaces in the following way:

$$
B=B^{\prime}+B^{\prime \prime}, \quad B^{\prime}=Q B, \quad B^{\prime \prime}=R B .
$$

Let us consider the finite-difference Liouville's Eq. (A.1) for the vector of fluctuations of a Brownian particle position

$$
\frac{\Delta}{\Delta t} \mathbf{B}_{m+k}^{m}(t)=i \hat{\mathscr{L}}(t, \tau) \mathbf{B}_{m+k}^{m}(t) .
$$

Modifying the last equation by operators $Q$ and $R$ successfully, we can split the dynamic equation (25) into two interconnected equations in the two mutually supplementary Euclidean subspaces

$$
\begin{aligned}
\frac{\Delta}{\Delta t} \mathbf{B}^{\prime}(t) & =i Q \hat{\mathscr{L}}(Q+R) \mathbf{B}(t)=i \hat{\mathscr{L}}_{11} \mathbf{B}^{\prime}(t)+i \hat{\mathscr{L}}_{12} \mathbf{B}^{\prime \prime}(t), \\
\frac{\Delta}{\Delta t} \mathbf{B}^{\prime \prime}(t) & =i R \hat{\mathscr{L}}(Q+R) \mathbf{B}(t)=i \hat{\mathscr{L}}_{21} \mathbf{B}^{\prime}(t)+i \hat{\mathscr{L}}_{22} \mathbf{B}^{\prime \prime}(t) .
\end{aligned}
$$

In the above equations the matrix elements $\hat{\mathscr{L}}_{i j}$ of quasi-operator $\hat{\mathscr{L}}$ have been introduced

$$
\begin{aligned}
& \hat{\mathscr{L}}_{11}=Q \hat{\mathscr{L}} Q, \quad \hat{\mathscr{L}}_{12}=Q \hat{\mathscr{L}} R, \quad \hat{\mathscr{L}}_{21}=R \hat{\mathscr{L}} Q, \quad \hat{\mathscr{L}}_{22}=R \hat{\mathscr{L}} R, \\
& \hat{\mathscr{L}}=\left(\begin{array}{ll}
\hat{\mathscr{L}}_{11} & \hat{\mathscr{L}}_{12} \\
\hat{\mathscr{L}}_{21} & \hat{\mathscr{L}}_{22}
\end{array}\right) .
\end{aligned}
$$

Operators $\hat{\mathscr{L}}_{i j}$ act as follows: $\hat{\mathscr{L}}_{11}$-from subspaces $B^{\prime}$ to $B^{\prime} ; \hat{\mathscr{L}}_{12}$-from $B^{\prime \prime}$ to $B^{\prime}$; $\hat{\mathscr{L}}_{21}$-from $B^{\prime}$ to $B^{\prime \prime}$; and $\hat{\mathscr{L}}_{22}$-from $B^{\prime \prime}$ to $B^{\prime \prime}$.

To simplify Liouville Eqs. (26) and (27) we exclude the irrelevant part $B^{\prime \prime}(t)$ and construct the closed equation for the relevant part $B^{\prime}(t)$. For this purpose it is 
necessary to obtain a step-by-step solution of Eq. (27)

$$
\begin{aligned}
\frac{\Delta \mathbf{B}^{\prime \prime}(t)}{\Delta t} & =\frac{\mathbf{B}^{\prime \prime}(t+\tau)-\mathbf{B}^{\prime \prime}(t)}{\tau}=i \hat{\mathscr{L}}_{21} \mathbf{B}^{\prime}(t)+i \hat{\mathscr{L}}_{22} \mathbf{B}^{\prime \prime}(t), \\
\mathbf{B}^{\prime \prime}(t+\tau) & =\mathbf{B}^{\prime \prime}(t)+i \tau \hat{\mathscr{L}}_{21} \mathbf{B}^{\prime}(t)+i \tau \hat{\mathscr{L}}_{22} \mathbf{B}^{\prime \prime}(t), \\
& =\left(1+i \tau \hat{\mathscr{L}}_{22}\right) \mathbf{B}^{\prime \prime}(t)+i \tau \hat{\mathscr{L}}_{21} \mathbf{B}^{\prime}(t), \\
& =U_{22}(t+\tau, t) \mathbf{B}^{\prime \prime}(t)+i \tau \hat{\mathscr{L}}_{21}(t+\tau, t) \mathbf{B}^{\prime}(t),
\end{aligned}
$$

where $U_{22}(t+\tau, t)=1+i \tau \hat{\mathscr{L}}_{22}(t+\tau, t)$ is the operator of a time step shift.

With the help of Eq. (30) we can derive the following expression for the next time step:

$$
\begin{aligned}
\mathbf{B}^{\prime \prime}(t+2 \tau)= & U_{22}(t+2 \tau, t+\tau) \mathbf{B}^{\prime \prime}(t+\tau)+i \tau \hat{\mathscr{L}}_{21}(t+2 \tau, t+\tau) \mathbf{B}^{\prime}(t+\tau) \\
= & U_{22}(t+2 \tau, t+\tau)\left[U_{22}(t+\tau, t) \mathbf{B}^{\prime \prime}(t)+i \tau \hat{\mathscr{L}}_{21}(t+\tau, t) \mathbf{B}^{\prime}(t)\right] \\
& +i \tau \hat{\mathscr{L}}_{21}(t+2 \tau, t+\tau) \mathbf{B}^{\prime}(t+\tau) \\
= & U_{22}(t+2 \tau, t+\tau) U_{22}(t+\tau, t) \mathbf{B}^{\prime \prime}(t)+i \tau\left[U_{22}(t+2 \tau, t+\tau)\right. \\
& \left.\times \hat{\mathscr{L}}_{21}(t+\tau, t) \mathbf{B}^{\prime}(t)+\hat{\mathscr{L}}_{21}(t+2 \tau, t+\tau) \mathbf{B}^{\prime}(t+\tau)\right] .
\end{aligned}
$$

Generalizing this result in case of the $m$ th discrete step we find the following final result:

$$
\begin{aligned}
\mathbf{B}^{\prime \prime}(t+m \tau)= & \left\{\hat{T} \prod_{j=0}^{m-1} U_{22}(t+(j+1) \tau, t+j \tau)\right\} \mathbf{B}^{\prime \prime}(t) \\
& +i \tau \sum_{j=0}^{m-1}\left\{\hat{T} \prod_{j^{\prime}=j}^{m-2} U_{22}\left(t+\left(j^{\prime}+2\right) \tau, t+\left(j^{\prime}+1\right) \tau\right)\right\} \\
& \times \hat{\mathscr{L}}(t+(j+1) \tau, t+j \tau) \mathbf{B}^{\prime}(t+j \tau) .
\end{aligned}
$$

Here $\hat{T}$ denotes the Dyson operator of chronological ordering. Substituting the irrelevant part of Eq. (26) into the right-hand side of Eq. (32), we obtain the closed finite-difference equation for the relevant part of the correlation state vector

$$
\begin{aligned}
\frac{\Delta}{\Delta t} \mathbf{B}^{\prime}(t+m \tau) \\
=i \hat{\mathscr{L}}_{11}(t+(m+1) \tau, t+m \tau) \mathbf{B}^{\prime}(t+m \tau)+i \hat{\mathscr{L}}_{12}(t+(m+1) \tau, t+m \tau) \\
\quad \times\left(\left\{\hat{T} \prod_{j=0}^{m-1} U_{22}(t+(j+1) \tau, t+j \tau)\right\} \mathbf{B}^{\prime \prime}(t)\right. \\
\quad-\tau \sum_{j=0}^{m-1}\left\{\hat{T} \prod_{j^{\prime}=j}^{m-2} U_{22}\left(t+\left(j^{\prime}+2\right) \tau, t+\left(j^{\prime}+1\right) \tau\right)\right\} \\
\left.\quad \times \hat{\mathscr{L}}_{21}(t+(j+1) \tau, t+j \tau) \mathbf{B}^{\prime}(t+j \tau)\right) .
\end{aligned}
$$


Substituting Eqs. (22) and (24) into Eq. (33), we derive a finite-difference kinetic equation of a non-Markov type for TCF $b(t)$

$$
\frac{\Delta b(t)}{\Delta t}=\lambda_{1} b(t)-\tau \Lambda_{1} \sum_{j=0}^{m-1} M_{1}(t-j \tau) b(j \tau) .
$$

Here the TCF $M_{1}(t)$ is the first-order memory function

$$
M_{1}(t-j \tau)=\frac{\left\langle\mathbf{W}_{1}(0) \mathbf{W}_{1}(t-j \tau)\right\rangle}{\left|\mathbf{W}_{1}(0)\right|^{2}} .
$$

Here $\Lambda_{1}$ is the frequency relaxation parameter of the first order with a square frequency dimension, and is defined by Eq. (9). Then if the dynamic variable $\mathbf{W}_{0}=$ $\mathbf{B}_{k}^{0}(0)$ represents the fluctuations of a Brownian particle position, the dynamic variable $\mathbf{W}_{1}=i \hat{\mathscr{L}} \mathbf{W}_{0}-\lambda_{1} \mathbf{W}_{0}$ contains fluctuations of pulses of a Brownian particle (see, Eqs. (10)). The function $M_{1}(t)$ is a time correlation function of fluctuations of a Brownian particle velocity.

Defining the corresponding projection operators to new dynamic variable $\mathbf{W}_{1}$ and repeating the above-described procedure, we find the finite-difference kinetic equation for $M_{1}(t)$

$$
\frac{\Delta M_{1}(t)}{\Delta t}=\lambda_{2} M_{1}(t)-\tau \Lambda_{2} \sum_{j=0}^{m-1} M_{2}(t-j \tau) M_{1}(j \tau) .
$$

Here $\lambda_{2}$ and $\Lambda_{2}$ are frequency relaxation parameters of the second order, $M_{2}(t)$ is the second-order memory function or memory function of the velocity correlation function for a Brownian particle (memory friction) [16-19]. In fact, Eq. (36) is a discrete finite-difference generalized Langevin equation (GLE). Thus, $M_{2}(t)$ can be associated with TCF of the stochastic Langevin forces, for which a similar equation can be obtained:

$$
\frac{\Delta M_{2}(t)}{\Delta t}=\lambda_{3} M_{2}(t)-\tau \Lambda_{3} \sum_{j=0}^{m-1} M_{3}(t-j \tau) M_{2}(j \tau)
$$

with the third-order frequency relaxation parameters $\lambda_{3}$ and $\Lambda_{3}$, and the memory function of the third order $M_{3}(t)$, respectively.

The three Eqs. (34), (36) and (37) are the exact consequence of microscopic discrete finite-difference equations of motion. Calculation of memory function $M_{i}(t)$ and the relaxation frequency parameters $\lambda_{j}, \Lambda_{k}$ are the central point here.

In case of a Brownian particle presented above, dynamic variables $\mathbf{W}_{0}$ and $\mathbf{W}_{1}$ are the position and pulses of random particles. In fact, they can be taken as characteristics of any other nonstationary process. The averaging operator of sampling with length $M$ elements, $\hat{A}=1 / M \sum_{j=0}^{M-1}$ (see Eq. (15)), applied to the intermediate local sampling can be changed by any other operator, depending on the goal of the investigation. The operator $\hat{A}$ allows to obtain clear or sharp fluctuations in the initial sampling of data $X(t)$ and to replace it by another $Y\left(t^{\prime}\right): X(t) \rightarrow Y\left(t^{\prime}\right)$, which contains the results of coarse-graining averaging. However, it is not always 
convenient to average a local sampling. In these cases the operator $\hat{A}$ can be replaced by another operator, which allows to obtain only one number from every local sampling. In the general case, another, more universal method of transformation of the initial sampling into the sampling with some specified (required) characteristics can become discrete wavelet-transform [21-25], which is defined by the following equation:

$$
W(j, k)=\sum_{j} \sum_{k} X(k) 2^{-j-2} \Psi\left(2^{-j} n-k\right) .
$$

Here $X(k)$ is the sampling (1) and $\Psi(t)$ is a time function with fast decay called mother wavelet. The following analysis can be applied to the new transformed data $W(j, k)$ according to the algorithm described above. Namely, memory functions $M_{i}(t)$ and frequency relaxation parameters $\lambda_{i}$ and $\Lambda_{i}$ can be calculated for the transformed data.

\subsubsection{Analog of Green-Kubo relation for diffusion coefficient for time discrete system}

According to the theory of random walks the mean-square displacements of a Brownian particle can be defined as

$$
\left\langle\Delta y^{2}\right\rangle_{t}=\int_{-\infty}^{+\infty} \Delta y^{2} \Phi_{1}(y, t) \mathrm{d} y=2 D t \quad(t \rightarrow \infty) .
$$

Here $\Phi_{1}(y, t)$ is the density of probability of a particle being at point $y$ at time moment $t$. Eq. (39) is a well-known Einstein relation for continuous displacements.

According to Eq. (39) the displacement during time $t$ is

$$
\Delta y_{t}=y(t)-y(0) \text {. }
$$

In case of the discrete time Eq. (40) can be rewritten in the form

$$
\Delta y(t)=\Delta y(T+j \tau)=y(T+(j+m) \tau)-y(T+j \tau), \quad t=m \tau, \quad m \gg 1 .
$$

Then the mean-square displacement of a Brownian particle is

$$
\begin{aligned}
& \left\langle\Delta y^{2}(T+(j+m) \tau)\right\rangle=\frac{1}{N-m} \sum_{j=0}^{N-m-1}[y(T+(j+m) \tau)-y(T+j \tau)]^{2}, \\
& \quad N>m \gg 1
\end{aligned}
$$

where $N-m$ is the quantity of "possible ways". The diffusion coefficient takes the following form:

$$
\begin{aligned}
& D=\frac{1}{2 m(N-m) \tau} \sum_{j=0}^{N-m-1}[y(T+(j+m) \tau)-y(T+j \tau)]^{2}, \\
& \text { at } N>m \gg 1(\text { or } t \rightarrow \infty) .
\end{aligned}
$$


Let us consider separately the sum in the last expression in terms of the velocity (see Eq. (17))

$$
\begin{aligned}
& \sum_{j=0}^{N-m-1}[y(T+(j+m) \tau)-y(T+j \tau)]^{2} \\
= & \underbrace{[y(T+m \tau)-y(T)]^{2}+[y(T+(1+m) \tau)-y(T+\tau)]^{2}+\cdots+[y(T+(N-1) \tau)-y(T+(N-m-1) \tau)]^{2}}_{(N-m) \text { of square brackets }[\cdots]} \\
= & {[y(T+m \tau)-y(T+(m-1) \tau)+y(T+(m-1) \tau)-\cdots-y(T)]^{2}+\cdots } \\
& +[y(T+(N-1) \tau)-y(T+(N-2) \tau)+y(T+(N-2) \tau)-\cdots \\
& -y(T+(N-m-1) \tau)]^{2} \\
= & \tau^{2}[v(T+(m-1) \tau)+v(T+(m-2) \tau)+\cdots+v(T)]^{2}+\cdots \\
& +\tau^{2}[v(T+(N-2) \tau)+v(T+(N-3) \tau)+\cdots+v(T+(N-m-1) \tau)]^{2} \\
& =\tau^{2} \sum_{j=0}^{N-m-1}\left[\sum_{j=0}^{m-1+j} v(T+k \tau)\right]^{2} .
\end{aligned}
$$

Then the expression for the diffusion coefficient (43) can be rewritten as

$$
D=\frac{\tau}{2 m(N-m)} \sum_{j=0}^{N-m-1}\left[\sum_{k=j}^{m-1+j} v(T+k \tau)\right]^{2} .
$$

Eq. (45) is the discrete finite-difference analog of the famous Green-Kubo relation for the diffusion coefficient. The given relation has been obtained from Einstein equation (39) for a discrete system. The asymptotic limit $t \rightarrow \infty$ can be replaced here by the similar condition $N \rightarrow \infty, m \rightarrow \infty, N>m$.

\section{Local noisy parameters}

Seismic data represent discrete random series, which is a recording of displacements of the Earth's surface. Therefore, we can use the above-stated formalism to analyze seismic data.

In particular, the local dependence of various characteristics [1,2] can serve as an additional source of information on properties of objects. Noise parameters $\lambda_{i}$ and $\Lambda_{i}$ are very sensitive to the presence of a nonrandom component in the sampling. The change in the character of the correlated noise and the appearance of the additional signal in the sampling can cause an alternation of these parameters. Hence, the time behavior of the local parameters $\lambda_{i}, \Lambda_{i}$ is important and informative when analyzing seismic data.

The procedure of localization consists of the following. Let us assume that we have an array of data $\left\{x_{1}, x_{2}, x_{3}, \ldots, x_{M}, \ldots, x_{N}\right\}$. Let us take the initial sampling of the fixed length $M$. Then by passing through all array of values with the "work window" of the fixed length $M$ we can calculate the time series of the noisy parameters $\left\{\lambda_{i}(T, T+M \tau), \lambda_{i}(T+\tau, T+(M+1) \tau), \ldots, \lambda_{i}(T+(N-M-1) \tau, T+\right.$ 
$(N-1) \tau)\}$ and $\left\{\Lambda_{i}(T, T+M \tau), \Lambda_{i}(T+\tau, T+(M+1) \tau), \ldots, \Lambda_{i}(T+(N-M-1) \tau\right.$, $T+(N-1) \tau)\}$.

Obviously, it is inadmissible to use both very large intervals (1000 and more points) and very short intervals (50 points and less) for the definition of local parameters $\lambda_{i}(t), \Lambda_{i}(t)$. In the first case the physical sense of the localization procedure is lost. On the other hand, it is impossible to carry out any plausible correlation analysis with small intervals because of gross errors. Therefore there is a necessity to find the optimum length of the initial local interval $(T, T+\tau, T+$ $2 \tau, \ldots, T+(M-1) \tau)$ or quantity $M$.

To determine the optimal minimal local sampling we have used the data corresponding to the calm state of the Earth before the technogenic explosion (an underground nuclear explosion). The calculation procedure consists in the following. We have taken the interval of 40 points as the starting point and have calculated all the noise low-order parameters $\lambda_{i}(i=1,2,3)$ and $\Lambda_{j}(j=1,2)$ by Eqs. (9) and (10). Then the interval was consistently increased by unit time segment $\tau$ and the relaxation parameters $\lambda_{i}, \Lambda_{j}$ were calculated every time at the increase of the interval. As a result of this procedure executed for the calm state of the Earth we have established that all parameters take "steady" numerical values at the interval approximately equal to 150 points and more (see Fig. 1 for more details). Namely, from Fig. 1 one can see that parameters $\lambda_{1}$ and $\Lambda_{1}$ take minimal values in their absolute quantity at this length of interval; the amplitude of value fluctuations of the parameter $\Lambda_{2}$ becomes lower and levels off. It is again an evidence of reduction of the noise influence. Fluctuations of parameters $\lambda_{2}$ and $\lambda_{3}$ also decrease and the parameters themselves take steady values starting with the sampling of length $\sim 150$ points.

Applying this procedure to other data of the calm state of the Earth, we find the same behavior of noise parameters $\lambda_{i}, \Lambda_{i}$ and detect the minimal interval of 150 points again. Thus, we choose the interval of such length as being optimal for accumulation of local statistics. ${ }^{1}$

\section{Definition of relaxation parameters $\lambda_{i}(t)$ and $\Lambda_{j}(t)$ for earthquakes and technogenic explosions data}

It is well known that modern seismic devices allow to derive different quantitative and qualitative data about the seismic state of the Earth. In this work we analyze three various weak local earthquakes (EQ's) in Jordan (1998) (EQ(1), EQ(2), EQ(3)), one strong earthquake in Turkey (summer 1999) [EQ(T)] and three local underground technogenic explosions (TE) (TE(1), TE(2), TE(3)) with the length of registration from 10000 to 25000 points. In case of strong EQ its seismogram contains 65000 registered points. All these experimental data were courteously given by the Laboratory of Geophysics and Seismology (Amman, Jordan). All data

\footnotetext{
${ }^{1}$ It should be noted the interest observation that local interval with 100-120 points was also found in previous work [2] devoted to seismic data analysis with the help of local Herst exponent.
} 

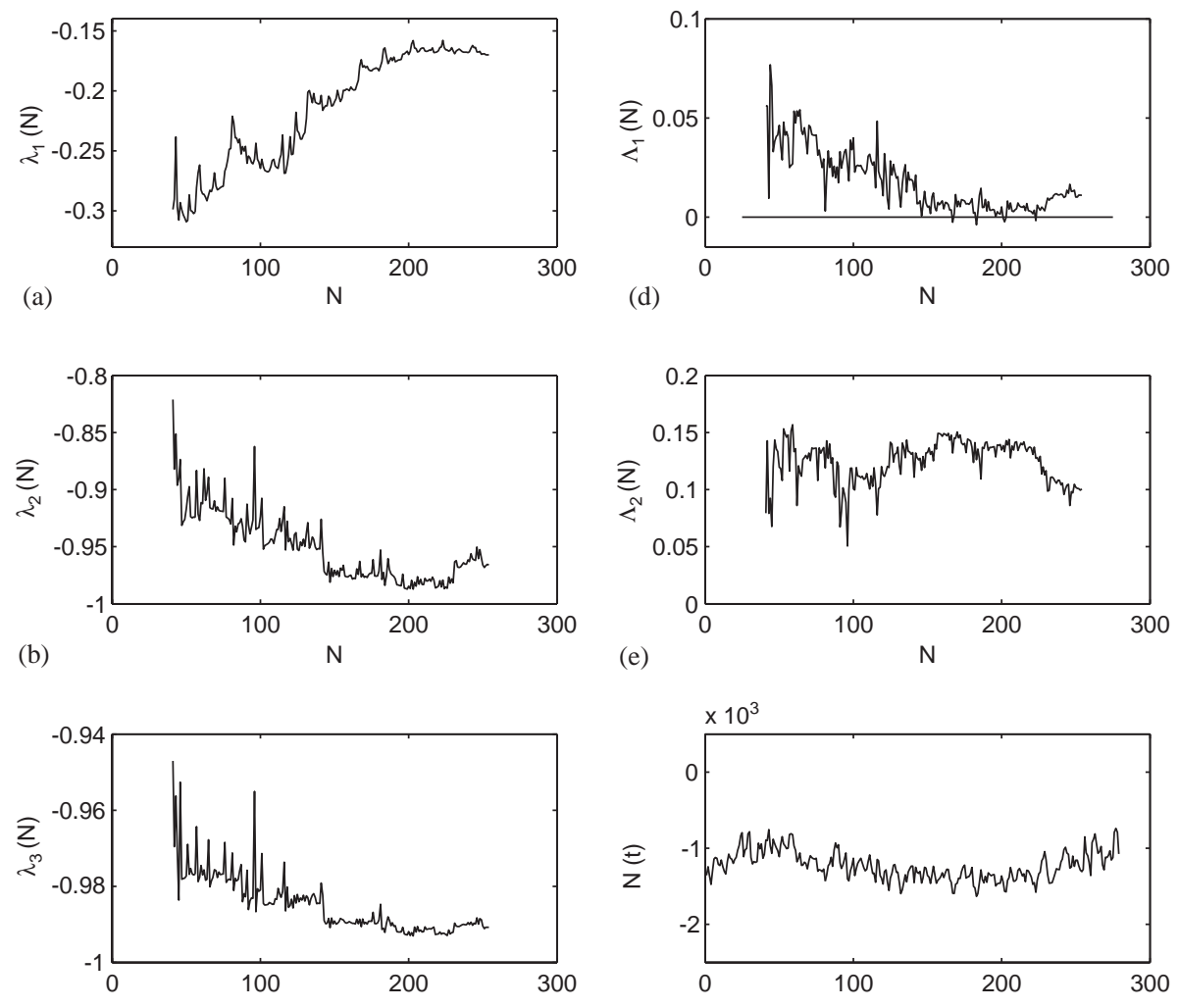

(c)

N

(f)

$t[\tau]$

Fig. 1. The definition of the optimal size for a local "working window". Calculations were carried out for the data of a steady state of the Earth. As a result we find that size $N \sim 150$ points is more optimal. The relative stability of all parameters is observed. Inset (f) shows seismic signals for the Earth's steady state (before underground TE).

correspond to transverse seismic displacements. The real temporal step of digitization $\tau$ between the registered points of seismic activity has the following values, viz, $\tau=0.02 \mathrm{~s}$ for the $\operatorname{EQ}(\mathrm{T})$, and $\tau=0.01 \mathrm{~s}$ for all other cases. We defined such characteristics as the maximal amplitude of signal fluctuations before "event" $a_{1}$, the maximal amplitude of signal fluctuations during "event" $a_{2}$, EQ (TE) power $a_{2} / a_{1}$, time interval till EQ (TE) $T_{0}$, continuance of "event" $T_{l}$ and finally the total time of signal recording $T_{\text {total }}$ directly from seismic data. These quantities are presented in Table 1. They give a clear notion about the duration and power of investigated phenomena. One can see from Table 1, that approximately 4500-5700 points are accounted for the visible part of a wavelet. This number of recorded points allows one to calculate and analyze local parameters $\lambda_{i}(t)$ and $\Lambda_{j}(t)$ in detail from Eqs. (9) and (10).

Let us describe the procedure of calculation of time dependence for $\lambda_{i}(t)$ and $\Lambda_{j}(t)$ in detail. It is based on the following operations. An interval with $M \sim 150$ points is 
Table 1

Some characteristics of technogenic explosions (TE(1), TE(2), TE(3)) and earthquakes (EQ(1), EQ(2), $\mathrm{EQ}(3), \mathrm{EQ}(\mathrm{T}))$ obtained from seismic data: $a_{1}$ is a maximal amplitude of signal oscillations before the "event", $a_{2}$ is maximal amplitude of signal oscillations at the time of the "event", $T_{0}$ is time from the beginning of the signal registration to the beginning of the "event", $T_{l}$ is the "event" duration, and $T_{\text {total }}=N$ is the total time of signal recording

\begin{tabular}{lllllll}
\hline & $a_{1}$ & $a_{2}$ & $a_{2} / a_{1}$ & $T_{0}$ & $T_{l}$ & $T_{\text {total }}$ \\
\hline $\mathrm{TE}(1)$ & $1.03 \times 10^{-3}$ & $8.24 \times 10^{-3}$ & 8 & 4091 & 4500 & 12500 \\
$\mathrm{TE}(2)$ & $1.5 \times 10^{-3}$ & $18.5 \times 10^{-3}$ & $12.3(3)$ & 3538 & 4500 & 10000 \\
$\mathrm{TE}(3)$ & $1.18 \times 10^{-3}$ & $56.5 \times 10^{-3}$ & 47.88 & 4091 & 4850 & 15000 \\
$\mathrm{EQ}(1)$ & $0.74 \times 10^{-3}$ & $7.1 \times 10^{-3}$ & 9.59 & 5682 & 4500 & 15000 \\
$\mathrm{EQ}(2)$ & $0.94 \times 10^{-3}$ & $6.1 \times 10^{-3}$ & 6.49 & 12308 & 5770 & 25000 \\
$\mathrm{EQ}(3)$ & $0.59 \times 10^{-3}$ & $14.1 \times 10^{-3}$ & 23.9 & 3182 & 5700 & 12500 \\
$\mathrm{EQ}(\mathrm{T})$ & $9 \times 10^{-3}$ & 20 & $2.2(2) \times 10^{3}$ & $\approx 13500$ & - & 65000 \\
\hline
\end{tabular}

taken, and noisy parameters $\lambda_{i}, \Lambda_{j}$ are calculated for it with the help of Eqs. (9) and (10). Then the operation of "stepwise shift to the right" at the interval of the fixed length $M$ is executed, and parameters are computed again. These actions are executed, while the initial sampling $X(t)$ will not be finished. As a result we obtain the following dependencies: $\quad\left\{\lambda_{i}(T, T+M \tau), \lambda_{i}(T+\tau, T+(M+1) \tau), \ldots, \lambda_{i}(T+\right.$ $(N-M-1) \tau, T+(N-1) \tau)\} \quad$ and $\quad\left\{\Lambda_{i}(T, T+M \tau), \Lambda_{i}(T+\tau, T+(M+1) \tau), \ldots\right.$, $\left.\Lambda_{i}(T+(N-M-1) \tau, T+(N-1) \tau)\right\}$. If the character of the noise in the investigated data changes, some signal will appear or disappear, and it will be directly reflected in the behavior of the relaxation characteristics.

The above-described procedure has been used to analyze EQ and TE data. The results are shown in Figs. 2, 3 and 4 and Table 2. However, in order to verify the optimized length of the local interval, we calculated local parameters $\lambda_{i}(t)$ and $\Lambda_{j}(t)$ ( $i=1,2,3),(j=1,2)$ at the local sampling with length $M=100,200,250,300,350$ and 400 points. It turned out that a large number of various noises are superimposed on the carrying trajectory at $M=100$ points in the behavior of $\lambda_{i}(t)$ and $\Lambda_{j}(t)$ (parameters has a gross errors). The line-shapes of $\lambda_{i}(t)$ and $\Lambda_{j}(t)$ practically cease to change at the sampling length $M=150$ and more $M=200,250, \ldots$. Once again it favors the view that the local interval with length $M=150$ points is optimal for the analysis of strong, weak EQs and TEs.

The detailed analysis of the received results allows to reveal the following features. Weak EQs and local underground TEs(for TE(3) and EQ(3), see Figs. 3 and 4):

(1) All the parameters $\lambda_{i}(t)$ take only negative values $\left(\lambda_{i}(t)<0\right)$, whereas noisy parameters $\Lambda_{j}(t)$ can be both positive and negative.

(2) Noisy parameter $\lambda_{1}(t)$ : Absolute magnitude $\left|\lambda_{1}\right|$ increases sharply in its amplitude by a factor approximately equal to 4-13.3 during EQ (various for different EQs), and then it returns to its initial state. Restoration time $T_{\lambda_{1}}$ and the duration of "event" $T_{l}$ are approximately equal for weak EQ, i.e., $T_{\lambda_{1}} \approx T_{l}$. Absolute magnitude $\left|\lambda_{1}\right|$ also exhibits an abrupt rise $\sim 2.2-3.5$ times higher for 

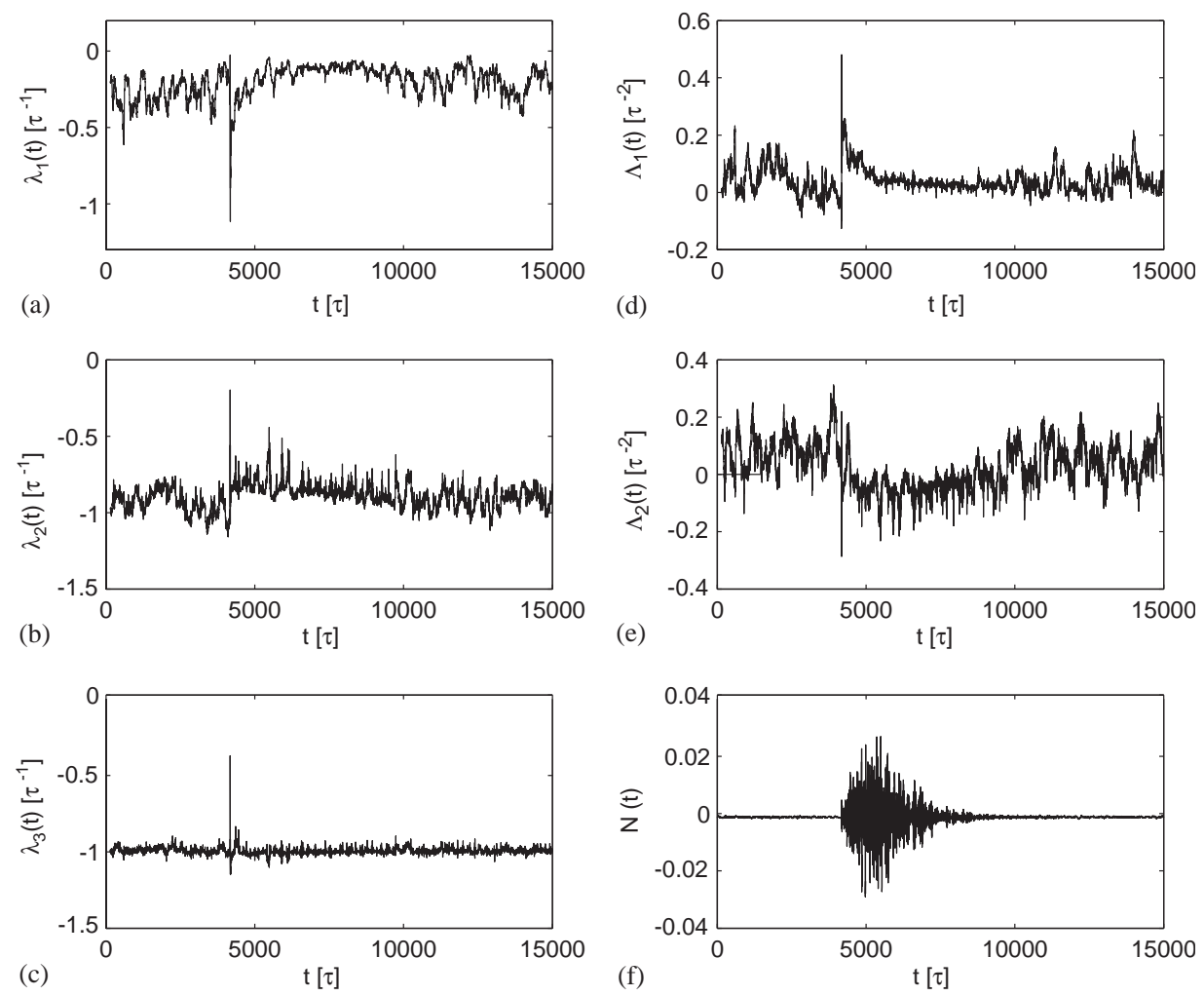

Fig. 2. The calculated time behavior of noisy relaxation parameters $\lambda_{i}$ and $\Lambda_{i}$ for the technogenic explosion TE(3) (a-e), whose signal is presented in inset (f).

TEs. However, it returns quickly to its normal level. The restoration time $\lambda_{1}(t)$ for TE is less than the duration of the "event" approximately by a factor of 2.5-3.

(3) Noisy parameter $\lambda_{2}(t)$ : Parameter $\lambda_{2}(t)$ responds to the beginning of the "event" by an abrupt rise of its value. It increases in amplitude both for TEs and for EQs. The character of the noise changes during the "event". The parameter responds to the power of the "event".

(4) Noisy parameter $\lambda_{3}(t)$ : This parameter always fluctuates close to its numerical value -1 , and shows an abrupt rise of its values at the beginning the "event" in the form of separated spikes (see Figs. 3 and 4). The parameter keenly responds to the noise changes.

(5) Noisy parameter $\Lambda_{1}(t)$ :It fluctuates near zero before and after the "event" changing its sign at this time. The parameter increases sharply at the "event" and always (!) remains positive. Then it decays smoothly. Restoration time $T_{\Lambda_{1}}$ and the duration of the "event" $T_{l}$ are approximately the same both for the EQ and TE. This parameter is very sensitive to the changes in the noise character. 

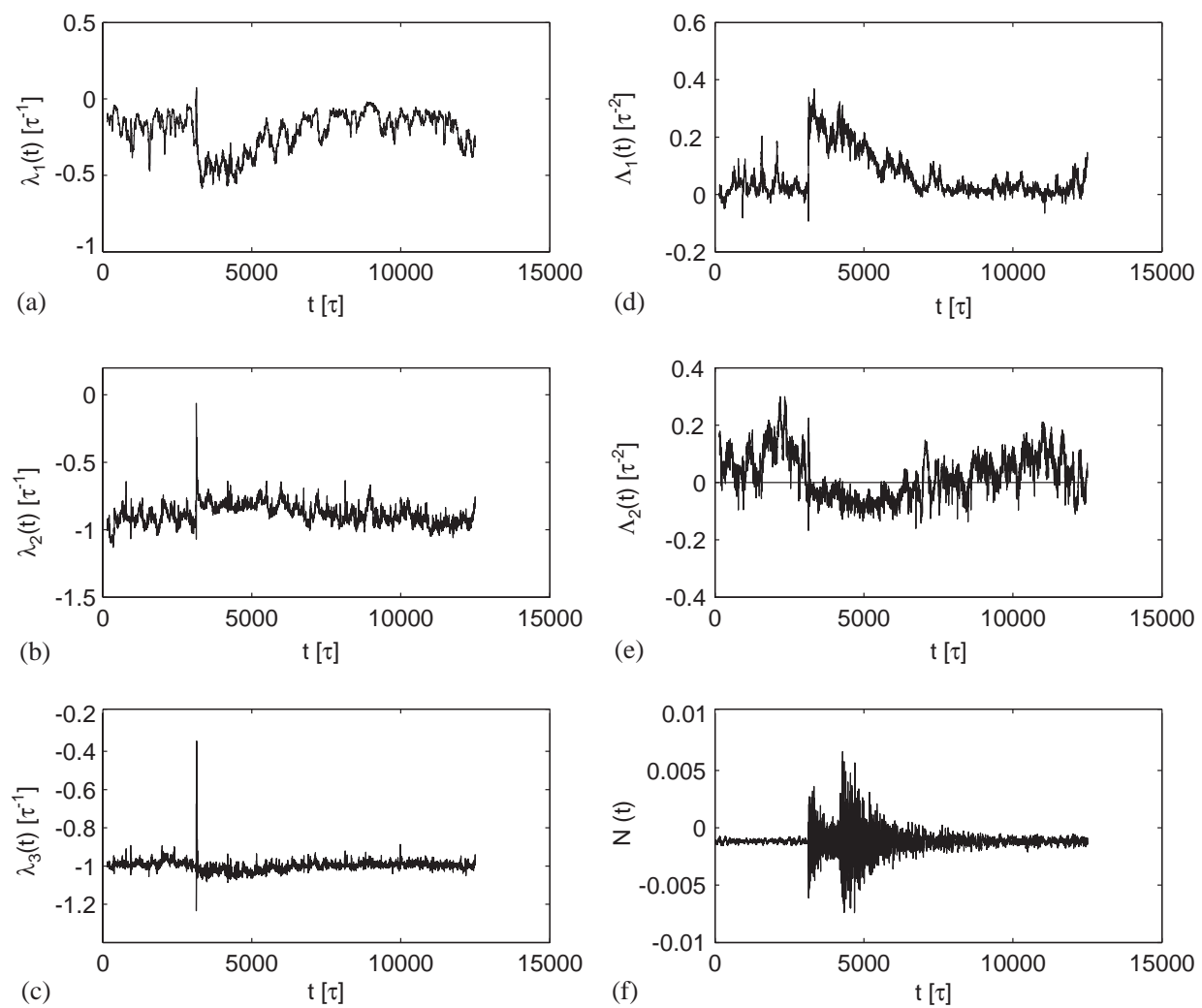

Fig. 3. The time behavior of noise relaxation parameters $\lambda_{i}$ and $\Lambda_{i}$ for the weak EQ(3) (see a-e), whose signal is also presented in inset (f).

For example, the data analysis of the $\mathrm{EQ}(2)$ shows that the separate burst of the amplitude values of $\Lambda_{1}(t)$ appears for $\approx 4000$ points up to the beginning of the $\mathrm{EQ}$, although such an indicator was not visually observed in the initial seismic data. ${ }^{2}$ It may be an evidence of high prognostic property of this parameter for EQs forecasting.

(6) Noisy parameter $\Lambda_{2}(t)$ : The noise changes of parameter $\Lambda_{2}(t)$ are observed during the "event" both during EQs and TEs. From Figs. 3 and 4 we can see that $\Lambda_{2}(t)$ has a distinctive negative depression during the "event".

Strong EQs (for $\mathrm{EQ}(\mathrm{T})$ see Fig. 5): ${ }^{3}$

\footnotetext{
${ }^{2}$ It turned out, that the noise variation is visible for all noise parameters $\lambda_{i}(t)$ and $\Lambda_{j}(t)(i=1-3 ; j=1,2)$ long before earthquake. However, it is more pronounced for time-dependence of the parameter $\Lambda_{1}(t)$.

${ }^{3}$ The strong Turkish earthquake presented here was analyzed on the basis of the data of different sources. However, we received always results, which were the same with presented in Fig. 4.
} 

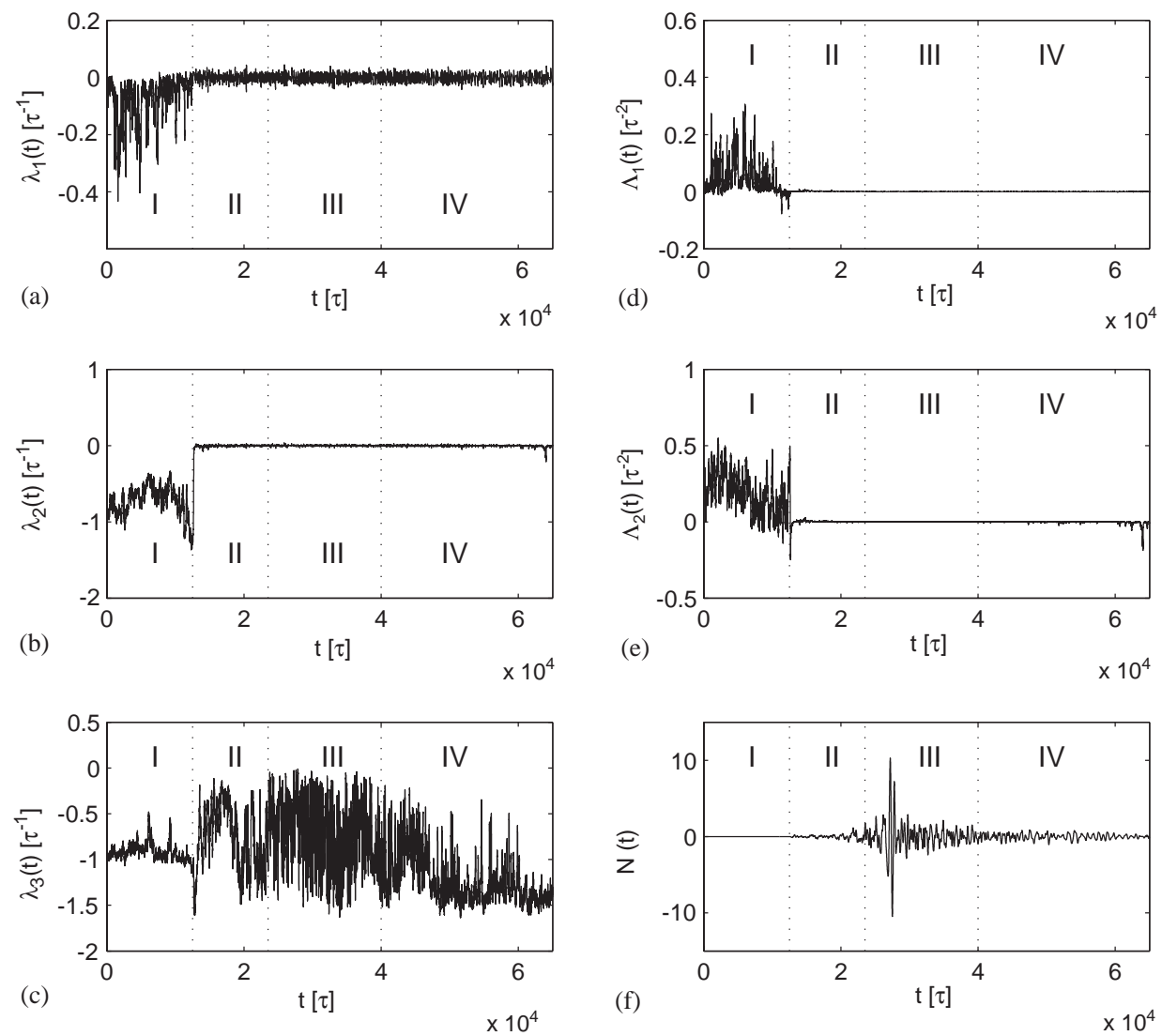

Fig. 4. The time behavior of the noisy relaxation parameters $\lambda_{i}$ and $\Lambda_{i}(\mathrm{a}-\mathrm{e})$ for the strong EQ(T), whose signal is presented in (f). The following regions: I- a calm state of the Earth's core, II - the state before the earthquake, III - the state during the earthquake and, finally, IV - the state after the event are divided by the vertical dotted lines.

Table 2

Characteristics of local parameters $\lambda_{1}(t)$ and $\Lambda_{1}(t): T_{\lambda_{1}}$ is the parameter $\lambda_{1}(t)$ relaxation time, $T_{\Lambda_{1}}$ is the relaxation time of $\Lambda_{1}(t), \tau_{\lambda}$ is the relaxation time for exponential attenuation of $\lambda_{1}(t)$, and $\tau_{\Lambda}$ is the relaxation time for exponential attenuation of $\Lambda_{1}(t)$

\begin{tabular}{llllllllllll}
\hline & $\begin{array}{l}\lambda_{0} \text { (units } \\
\text { of } \tau^{-1} \text { ) }\end{array}$ & $\begin{array}{l}\Delta \lambda \text { (units } \\
\text { of } \tau^{-1} \text { ) }\end{array}$ & $T_{\lambda}$ & $\tau_{\lambda}$ & $\begin{array}{l}\Lambda_{0} \text { (units } \\
\text { of } \tau^{-2} \text { ) }\end{array}$ & $\begin{array}{l}\Delta \Lambda \text { (units } \\
\text { of } \tau^{-2} \text { ) }\end{array}$ & $T_{\Lambda}$ & $\tau_{\Lambda}$ & $\Delta \lambda / \lambda_{0}$ & $\Delta \Lambda / \Lambda_{0}$ & $T_{l} / T_{\lambda}$ \\
\hline $\mathrm{TE}(1)$ & -0.13 & -0.425 & 1800 & 90 & 0.02 & 0.28 & 4500 & 45 & 3.27 & 14 & 2.5 \\
$\mathrm{TE}(2)$ & -0.15 & -0.34 & 1440 & 100 & 0.02 & 0.28 & 4032 & 55 & $2.26(6)$ & 14 & 3.125 \\
$\mathrm{TE}(3)$ & -0.15 & -0.33 & 1870 & 80 & 0.002 & 0.42 & 4850 & 45 & 2.2 & 210 & 2.59 \\
$\mathrm{EQ}(1)$ & -0.045 & -0.6 & 4500 & 170 & 0.005 & 0.43 & 4500 & 110 & $13.3(3)$ & 86 & 1 \\
$\mathrm{EQ}(2)$ & -0.1 & -0.5 & 5770 & 170 & 0.001 & 0.35 & 5770 & 130 & 5 & 350 & 1 \\
$\mathrm{EQ}(3)$ & -0.12 & -0.47 & 5700 & 210 & 0.01 & 0.35 & 5700 & 180 & $3.91(6)$ & 35 & 1 \\
\hline
\end{tabular}



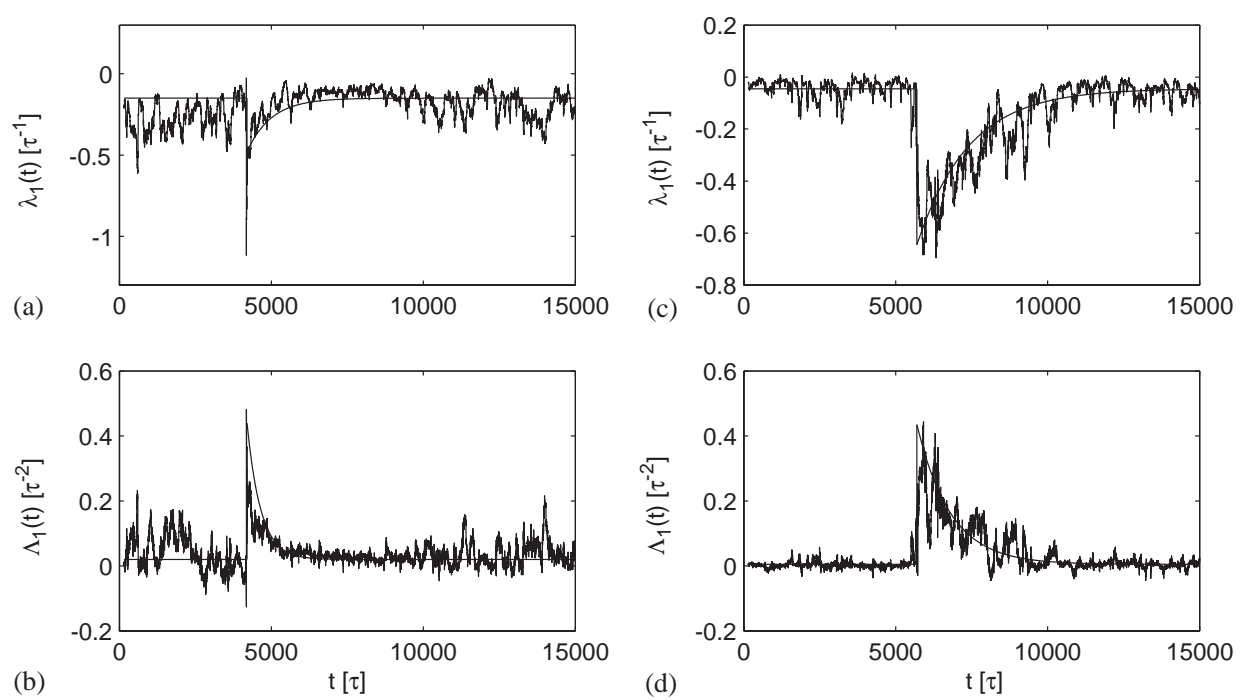

Fig. 5. The time behavior of the first two noisy relaxation parameters $\lambda_{1}, \Lambda_{1}$ for TE(3) (a and $b$ ) and for the weak EQ(1) (c and d). Solid lines show fitted functions (46) and (47) with corresponding parameters, presented in Table 2. One can note the typical exponential restoration of the parameters with the beginning of the event.

All parameters react keenly to the appearance of the signal in case of a strong EQ. Let us consider the behavior of the local parameters in this case in detail.

(7) Noisy parameter $\lambda_{1}(t)$ :The parameter demonstrates the presence of noise and takes negative values before the "event" (region $I$ in Fig. $5 \mathrm{f}$ ). As the "event" approaches, the amplitude of fluctuations decreases, and oscillations turn into negligible fluctuations close to zero value for II, III and IV Regions (for more details, see Fig. 5).

(8) Noisy parameter $\lambda_{2}(t)$ : A noise is also observed in the behavior of this parameter before the "event", and this parameter takes only negative values. However, its values decrease sharply in absolute magnitude and begin to take values close to zero with the appearance of an EQ signal (Fig. 5b).

(9) Noisy parameter $\lambda_{3}(t)$ : This parameter takes only negative values at all times $t$. The negligible noise appears before the "event". As the "event" approaches the noise increases in amplitude by the factor 3-5. The amplitude of oscillations begins to decrease in Region IV (see Fig. 5c).

(10) Noisy parameters $\Lambda_{1}(t)$ and $\Lambda_{2}(t)$ : Parameters fluctuate, taking positive values mainly before the "event" (Region I). When the "event" starts their values increase greatly in absolute magnitude approaching zero. At the beginning of Region II both parameters change their sign from positive to negative. Then for Regions II, III and IV we see only a right line with $\Lambda_{1}(t), \Lambda_{2}(t) \rightarrow 0$ on the scales of Figs. $5 \mathrm{~d}$ and e. However, the parameter $\Lambda_{2}(t)$ has faintly visible fluctuations for Region IV characterizing the final EQ phase (see Figs. 5d and e). 
Hence, all parameters are very sensitive to the approach of a strong EQ. A sharp change in their behavior is appreciable before strong fluctuations of the Earth's surface beyond 10000 points $(\sim 3.5 \mathrm{~min})$.

\section{Simple exponential model for time local behavior of noisy relaxation parameters $\lambda_{1}(t)$ and $\Lambda_{1}(t)$}

As can be seen from Figs. 3-5 all relaxation parameters are very sensitive to the beginning of EQ and/or a TE. The behavior of parameters $\lambda_{1}(t)$ and $\Lambda_{1}(t)$ in weak local EQs and local underground explosions TEs is of great interest. These parameters are initial in our calculations (see Eqs. (9), (10)). The analysis has shown that parameters oscillate near average values $\lambda_{0}$ and $\Lambda_{0}$, correspondingly, before and after the oscillations visible on seismograms. The results of the behavior of these parameters for the TE(3) and the EQ(3) are presented in Figs. 3a-d and Fig. 4a-d. However, a sudden rise by factors $\Delta \lambda_{0}$ and $\Delta \Lambda_{0}$ is always observed in the behavior of these parameters at the enhancement or the appearance of the signal (it is seen at the beginning of the EQ or the TE in seismogram data). Furthermore, continuous attenuation occurs. During all this time these parameters have a well-defined pronounced trend. Such behavior of $\lambda_{1}(t)$ and $\Lambda_{1}(t)$ gives us a possibility of modelling the time dependence of these parameters by some simple mathematical functions. The fitting procedure showed that the time behavior of these parameters can be well approximated by the following simple time dependence:

$$
\begin{aligned}
& \lambda_{1}(t)=\lambda_{0}+\Delta \lambda \cdot \exp \left\{-\frac{t-T_{0}}{T_{\lambda}}\right\} \cdot H\left(t-T_{0}\right), \\
& \Lambda_{1}(t)=\Lambda_{0}+\Delta \Lambda \cdot \exp \left\{-\frac{t-T_{0}}{T_{\Lambda}}\right\} \cdot H\left(t-T_{0}\right),
\end{aligned}
$$

where $H(t)$ is the Heaviside function, and $T_{\lambda}$ and $T_{\Lambda}$ are relaxation times of $\lambda_{1}(t)$ and $\Lambda_{1}(t)$, correspondingly. Time $T_{0}$ is the same in Eqs. (46) and (47) for parameters $\lambda_{1}(t)$ and $\Lambda_{1}(t)$. Its numerical values are presented in Table 1.

The numerical values of the variables, included in the last Eqs. (46), (47) were defined for our EQs and TEs by comparison of localization results with these equations. They are presented in Table 2 .

Therefore, it was proved that the restoration of these parameters to their steady values occurs according to the exponential law. As can be seen from Fig. 4 this description best suits $\lambda_{1}(t)$ and $\Lambda_{1}(t)$ of weak EQs. The foregoing estimations fully strengthen our resume (2) and (5) of Section 5.

The results presented in the last three columns of Table 2 might be of interest for readers of this paper. As quantities $\Delta \lambda_{0} / \lambda_{0}$ and $\Delta \Lambda_{0} / \Lambda_{0}$ show the rise of value of the corresponding parameter at the explosion (at the EQ), the ratio between the "event" duration $T_{l}$ and the relaxation time $T_{\lambda}$ discovers a remarkable distinction between TEs and weak EQs. 


\section{Conclusion}

In this work a universal method for investigating nonstationary, unsteady and non-Markov random processes in discrete systems is suggested. This universality is achieved by combining the opportunities of microscopic, mesoscopic and macroscopic descriptions for complex systems. This method allows to find and to analyze fast, slow and superslow processes. To investigate superslow processes we propose to use the model of "a quasi-Brownian particle" which moves chaotically in heat. The wavelet-transformation of the initial time series and generalized kinetic Langeven equation can be used for this purpose.

This method helps to analyze and differentiate similar signals of different origin. Theoretical investigations have been realized by means of two methods supplementing each other: the statistical theory of discrete non-Markov stochastic processes [2] and the local noisy parameters. The application of the last method gives a possibility to study nonstationary and unsteady processes with alternation and superimposition of different modes. The correlation between the time scales characteristic of different modes may be different. However, if accurately realized, the localization procedure of the parameters and the calculation of their dynamics allow, as a rule, to separate the noise and the signals [1,2], and to carry out their quantitative and informative analysis.

Another important advantage of the method is the possibility to operate it in a "real-time" regime, i.e., it can be put into practice immediately after obtaining the data, which is of great practical value.

The developed approach has been tested for strong and weak EQs data and nuclear underground TEs. As a result we have obtained the following results.

The time behavior of the local relaxation parameters can be described by simple model relaxation functions. The temporal relaxation of parameters $\lambda_{1}(t)$ and $\Lambda_{1}(t)$ in weak EQs and TEs after the beginning of the "event" occurs according to the exponential law. However, the restoration and duration of the events are practically the same in the case of weak EQs. The restoration time of parameters $\lambda_{1}(t)$ and $\Lambda_{1}(t)$ in the case of TEs differs noticeably from the duration of the event. Thus, this approach can be useful in recognition of these two different seismic phenomena. From the analysis of strong EQ data one can see that the behavior of all parameters changes greatly long before the EQ. For example, such a change for the EQ(T) presented here occurs $\sim 3.5 \mathrm{~min}$ before the main event. This change of $\lambda_{i}(t)$ and $\Lambda_{i}(t)$ obtained for strong EQs opens the possibility for a more accurate registration of the beginning of changes of the parameters before the visual wavelet and real EQs.

We are sure that the suggested method can be very useful for the study of a wide class of random discrete processes in real complex systems of live and of lifeless things: in cardiology, physiology, neurophysiology, biophysics of membranes and seismology.

\section{Acknowledgements}

We gratefully acknowledge Prof. Raoul Nigmatullin for stimulating discussion and Dr. L.O. Svirina for technical assistance. This work was supported in part by the 
Russian Fund for Basic Research (Grant No. 02-02-16146), Ministry of Education of RF (Grant No. 02-3.1-538) and RHSF (No. 03-06-00218a).

\section{Appendix A. Three forms of the quasi-operator $\hat{\mathscr{L}}$}

Equations of motion of a random variable $x$ with the use of the derivative of the three different forms [26] are represented here.

Using evolution operator, we can write the equation of motion for a discrete case as follows:

$$
\mathrm{d} x(t) / \mathrm{d} t=i \hat{\mathscr{L}} x(t) .
$$

However, there is a possibility of application of the time derivative $\mathrm{d} / \mathrm{d} t \rightarrow \Delta / \Delta t$ in three different forms:

(1) "Right" derivative (with decurrent difference in numerator)

$$
\frac{\Delta x(t)}{\Delta t}=\frac{x(t+\tau)-x(t)}{\tau}=\frac{1}{\tau} U(t+\tau, t) x(t)
$$

with Liouville's quasioperator of the following form:

$$
\hat{\mathscr{L}}(t, \tau)=-\frac{i}{\tau}[U(t+\tau, t)-1] ;
$$

(2) "Left" derivative (with ascending difference in numerator)

$$
\frac{\Delta x(t)}{\Delta t}=\frac{x(t)-x(t-\tau)}{\tau}=\frac{x(t)-U^{-1}(t, t-\tau) x(t)}{\tau}=\frac{1}{\tau}\left[1-U^{-1}(t, t-\tau)\right] x(t)
$$

with the Liouvillian of the following form:

$$
\hat{\mathscr{L}}(t, \tau)=-\frac{i}{\tau}\left[1-U^{-1}(t, t-\tau)\right]
$$

(3) "Central" derivative (with a central difference in the numerator)

$$
\begin{aligned}
\frac{\Delta x(t)}{\Delta t} & =\frac{x(t+\tau)-x(t-\tau)}{2 \tau}=\frac{x(t+\tau)-x(t)}{2 \tau}-\frac{x(t)-x(t-\tau)}{2 \tau} \\
& =\frac{1}{2 \tau}\left[U(t+\tau, t)-U^{-1}(t, t-\tau)\right] x(t) .
\end{aligned}
$$

Then the quasioperator $\hat{\mathscr{L}}$ takes the following form:

$$
\hat{\mathscr{L}}(t, \tau)=-\frac{i}{2 \tau}\left[U(t+\tau, t)-U^{-1}(t, t-\tau)\right]
$$

In the calculations and the analysis presented in this work we have used the derivative of the first form [see Eqs. (A.2), (A.3)]. 


\section{References}

[1] P.Ch. Ivanov, L.N. Amaral, A.L. Goldberger, Sh. Havlin, M.G. Rosenblum, H.E. Stanley, Z.R. Struzik, Chaos 11 (2001) 641.

[2] R. Yulmetyev, F. Gafarov, P. Hänggi, R. Nigmatulin, Sh. Kayumov, Phys. Rev. E 64 (2001) 066132.

[3] A. Sornette, D. Sornette, Tectonophysics 179 (1990) 327.

[4] G. Igarashi, Geophys. Res. Lett. 27 (2000) 1973.

[5] D. Sornette, C. Sammis, J. Phys. I France 5 (1995) 607.

[6] J.-C. Anifrani, C. Le Floc'h, D. Sornette, B. Souillard, J. Phys. I France 5 (1995) 631.

[7] C.G. Bufe, D.J. Varnes, J. Geophys. Res. 98 (1993) 9871.

[8] P. Bak, C. Tang, K. Wiesenfeld, Phys. Rev. A 38 (1989) 364;

P. Bak, C. Tang, J. Geophys. Res. 94 (1989) 15635.

[9] D. Sornette, L. Knopoff, Bull. Seismol. Soc. Am. 87 (1997) 789.

[10] R. Yulmetyev, P. Hänggi, F. Gafarov, Phys. Rev. E 65 (2002) 046107.

[11] R. Yulmetyev, P. Hänggi, F. Gafarov, Phys. Rev. E 62 (2000) 6178.

[12] R.M. Yulmetyev, A.V. Mokshin, P. Hänggi, V.Yu. Shurygin, Phys. Rev. E 64 (2001) 057101.

[13] T. Scopigno, U. Balucani, G. Ruocco, F. Sette, J. Phys.: Condens. Matter 12 (2000) 8009.

[14] H. Mori, Prog. Theor. Phys. 33 (1965) 423;

H. Mori, Prog. Theor. Phys. 34 (1965) 765.

[15] R. Zwanzig, Phys. Rev. 124 (1961) 1338;

R. Zwanzig, Ann. Rev. Phys. Chem. 16 (1965) 67;

R. Zwanzig, J. Chem. Phys. 3 (1960) 106.

[16] M.E. Tuckerman, B.J. Berne, J. Chem. Phys. 95 (1991) 4389.

[17] B.J. Berne, R. Pecora, Dynamic Light Scattering, Wiley-Interscience, New York, 1976.

[18] P. Hänggi, P. Talkner, M. Borkovec, Rev. Mod. Phys. 62 (1990) 250.

[19] J.T. Hynes, Theory of chemical reaction dynamics, in: M. Baer (Ed.), Chemical Rubber, CRC Press, Boca Raton, 1985, p. 171.

[20] C. Papenfuss, P. Ván, W. Muschik, cond-mat/0212095, 2003.

[21] I. Daubechies, Comm. Pure Appl. Math. 41 (1998) 906;

I. Daubechies, IEEE Trans. Inf. Theory 36 (1990) 961;

I. Daubechies, Ten Lectures on Wavelets, CBMS Lecture Notes Series, SIAM, Philadelphia, 1991.

[22] J.M. Combes, A. Grossmann, P. Tchamitchian (Eds.), Wavelets, Springer-Verlag, Berlin, 1989.

[23] R. Coifman (Ed.), Wavelets and Their Applications, Jones and Barlett Publ., Boston, 1992.

[24] C.K. Chui, An introduction to Wavelets (Wavelets Analysis and Its Applications), Vol.1, Academic Press Inc., San Diego, 1992.

[25] N.M. Astafieva, Uspehi Phiz. Nauk 166 (1996) 1145 (in Russian).

[26] G.A. Korn, T.M. Korn, Mathematical Handbook, McGraw-Hill, New York, 1968. 